\title{
Gait Processing and Performance using Angular Velocity and Leaping Angle Parameters
}

\author{
Ching Yee Yong, Rubita Sudirman, Kim Mey Chew
}

\begin{abstract}
Gait analysis is a process of learning the motion of human and animal by wearable sensor approach or/and vision approach. This analysis mainly used in medical and sports field where the study of body parts is crucial. 3-space sensor consists of accelerometer, gyroscope and compass sensors, built in one device. In this project, 3-space sensor was used to collect the data of walking and jogging motion, from test subjects performing those activities on a treadmill. Two components of motion, the angular velocity of the test subjects' arm and the angle of leaping motion were selected and investigated. The data were then analyzed and processed using MATLAB using Principal of Component Analysis (PCA). PCA was used in gait analysis due to its ability to combine and reduce the number of variables of the obtained data. This method able to ease the flow of analysis since the variables had been reduced. The function called "Quiver" was used in order to generate the vector for each point plotted in the graph for both motions. Lastly, the accuracy of the analyzing process $(70 \%$ for walking and $100 \%$ for jogging motions) will be used to create a system that can recognize the motion on any given data.
\end{abstract}

Index Terms: Motion clustering, angular velocity, leaping angle, PCA.

\section{INTRODUCTION}

GAIT analysis is a study about movement of animal, or animal locomotion, which is also implemented for analyzing human motions, and have been widely used in medical purpose. This is crucial to illustrate the different body dynamic which depends on the test subjects' conditions, as stated by [1].

There are two methods to investigate human motions, namely vision based approach by using video camera, for recording the movement of the subject and then processed by computer. The second method is wearable sensor based approach. This can be done by mounting a tri-axial sensor on the test subject's part of body, for generating sets of motion data. Data generated by both approaches will be processed using specific software in order to get the orientations (on 3-axis), rotation and acceleration (force) of a given motion [2].

\section{RESEARCH REVIEW}

The most common method used in gait analysis is the vision based method [3].

Revised Manuscript Received on June 22, 2019.

Ching Yee Yong, School of Engineering and Technology, University College of Technology Sarawak, 96000Sibu, Sarawak

Rubita Sudirman, School of Electrical Engineering, Faculty of Engineering, 81310 Skudai, Johor Bahru, Johor

Kim Mey Chew, School of Computing and Creative Media, University College of Technology Sarawak, 96000 Sibu, Sarawak
However, there are several disadvantages need to be taken in consideration for using this method such as cost for highend equipment, space for data collection and lighting aspect. Due to these shortcomings, many have tried in developing other alternative for gait analysis, which is wearable sensorbased approach. In this project, we will try to test the capability of wearable sensor-based approach for motion data collection and re-present the data in a way that understandable by everyone.

3 -space sensor was proposed in this project due to its cost. One sensor was mounted on the test subject and establish a wireless connection with the processor. The test subject is free to move for every motion such as running, jumping or walking without any restriction that normally happen for vision based due to the coverage area from the camera shooting angle. This method also can be done in outdoor condition, without considering any lighting factor. Therefore, this method is practical to be used in research related to sports science, which is more likely to be conducted in actual pitch. Theoretically, wearable sensorbased has more advantages if compared to vision based. However, the way of implementing should be welldeveloped to match the quality of output.

Human activity recognition can be done by using smartphone and motion sensor [4], while limb motion such as hand gesture recognition can be detected by using depth sensor [5] and motion controller [6].

Gait analysis is an important study of human locomotive which give a great advantage on sports science and medical. [7]used multiple wearable inertial sensors on upper limb motion tracking to give appropriate assessment to the stroke patients before they are going to be discharged from the hospital. [8] used wearable sensor in gait recognition experiment to recognize a particular individual. These are the applications used in the domain of biometric identification by using wearable sensor as data acquisition.

\section{MATERIALS AND METHODS}

\section{A. Data Collection}

The objective of this project is to differentiate the motion of test subjects. The two test subjects for this experiment are two male persons, with the age of 23 , healthy, and without medical record of defect or other sickness. The test subjects were mounted by a 3 -space sensor at their upper right arm, for jogging and walking activities. These motions were performed on a treadmill and the data were transmitted to the processor for further processing. 
Experiment was started by identifying the subjects' normal speed of jogging and walking. Test subjects were requested to perform jogging and walking activities on a treadmill for 10 meters long distance. The times for covering up the distance were stamped and the speeds for these activities were calculated. The jogging and walking speed were set to $6.5 \mathrm{ft} / \mathrm{s}$ and $3.7 \mathrm{ft} / \mathrm{s}$ respectively.

The test subjects were then being asked to walk on the treadmill for different distances with the pre-set speed. This process was repeated 5 times each for walking distance in $10 \mathrm{~m}, 20 \mathrm{~m}, 30 \mathrm{~m}, 40 \mathrm{~m}, 50 \mathrm{~m}, 100 \mathrm{~m}, 150 \mathrm{~m}, 200 \mathrm{~m}, 250$ $\mathrm{m}, 300 \mathrm{~m}$, and the whole process was repeated for jogging motion. At the end, there are 50 data each from two subjects for jogging and walking motion. The data logged by the sensor were then processed by MATLAB.

\section{B. Angular Velocity}

Arm and leg are coordinating with each other to perform jogging and walking motion. In this project, we cover only arm segment since one sensor was utilized. While moving forward, the subject is periodically swinging his arm forward and backward (rotation in y axis) and also rotation in vertical axis (z-axis), as shown in Figure 1.

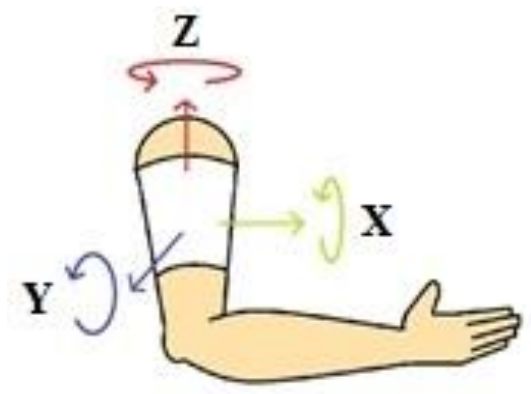

Fig. 1 Rotation and motion axis of subject's arm

Two variables were proposed to classify a motion, namely, angular velocity and leaping angle. MATLAB was used to compute these variables from each swing for classification. Furthermore, Principle Component Analysis (PCA) as shown in Figure 2 was used to categorize the data into their respective dimension by realigning the scatter plot of the data in order to get the actual axis of the arm's rotations perfectly along $\mathrm{y}$ and $\mathrm{z}$ axes.

After the graph was aligned by PCA, then all the positive angular velocity vectors were detected in $\mathrm{y}-\mathrm{z}$ plane, and the mean for the vectors was computed. Positive angular velocity vector was defined as the subject's arm swing in anti-clockwise direction, while the angle or rotation is positive.

This project covered only the positive angular velocity. In a complete cycle there are four types of motions, positive upward swing, positive downward swing, negative upward swing and negative downward swing as shown in Figure 3. All of the motions look similar, however, they are produced by different set of muscles and directions.

The next step was to calculate the mean of all positive swing detected during walking or jogging. Since the swings performed by the test subjects are not perfect with constant speed, thus average value for all the swings were computed.
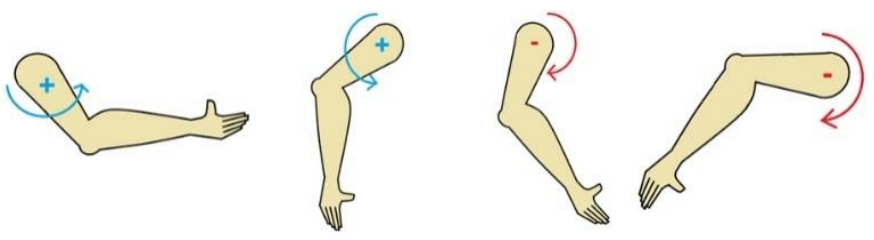

Fig.2 From left: positive upward swings, positive downward swings, negative downward swings, and negative upward swings

\section{Angle of Leaping Motion}

Based on [9], in order to complete a cycle of running or jogging motions, the runner shall be airborne twice, meaning to say, there will be at any instance where the test subjects leap in order to get airborne. For walking, it covers only two phases, which the test subjects are in a position called stance, with their weights are supported only by a foot, and another phase is when both feet touch the ground. Therefore, in walking motion, leaping motion does not exist. This significant difference between walking and jogging can be used to differentiate both motions and in this project, the angles of acceleration of the test subjects after leaping were used as the main component to distinguish both motions.

In the process to detect the angle of leaping motion, PCA was not utilized since the algorithm may affect the horizontal axis of the subjects when they are touching the ground. Based on Figure 1, $\mathrm{x}$ and $\mathrm{z}$ axes of the accelerometer sensor were considered for the leaping motion. In order to re-present the leaping motion from the accelerometer data, a function in MATLAB called quiver was used to plot the vector of the acceleration [10].

Figure 4 illustrated the walking motion vectors. The angle of the vectors were flatter if compared to jogging motion vectors as shown in Figure 5. Thus, it can be concluded that the jogging motion has significant change in vertical axis than the walking motion.

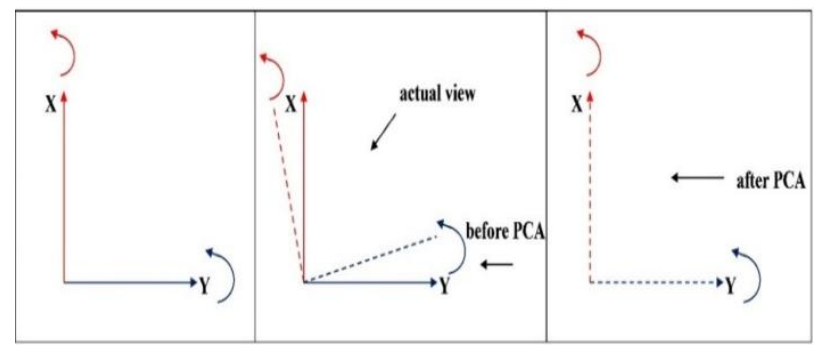

Fig. 3 Arm rotation axis from experiment (left), actual view (center), actual arm rotation axis rotated by PCA (right)

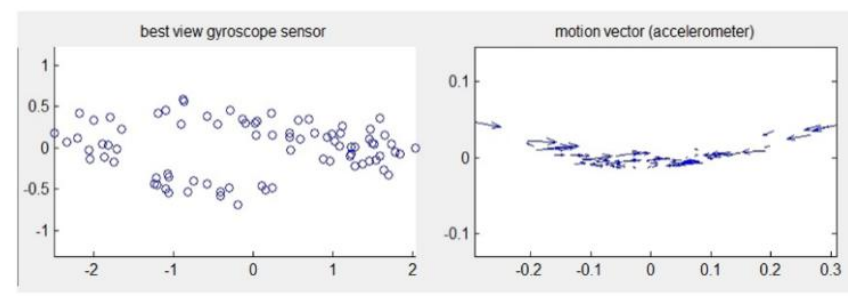

Fig. 4 Scatter plot of gyroscope data for walking motion (left), Vector plot of walking motion (right) 


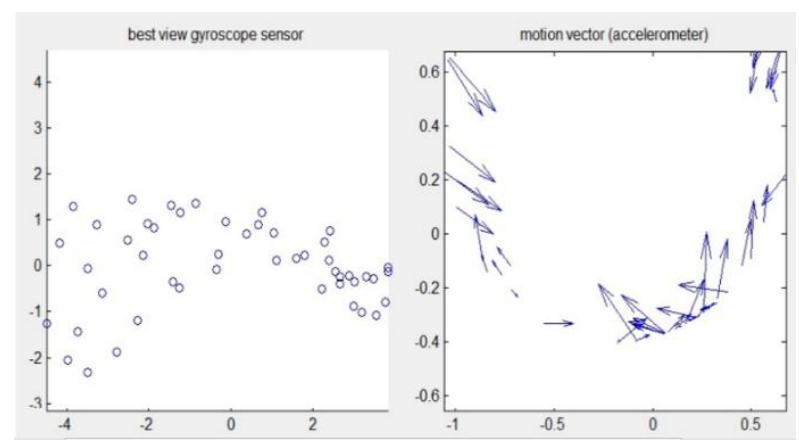

Fig. 5 Scatter plot of gyroscope data for jogging motion (left), Vector plot of jogging motion (right).

In order to generate the vector for all points in the scatter plot, the magnitude of resultant vector $(\vec{R})$ was computed by calculating, the difference in horizontal axis $(\vec{A})$ and vertical axis $(\vec{B})$ between two consecutive points in the scatter plot, as shown in Equation 2. While the angle of leaping motion when the subjects leap forward can be computed by using Equation 1, where $\theta$ can be calculated as a result of inverse tangent of $\mathrm{A}$ over $\mathrm{B}$.

$\tan \theta=\frac{B}{A}$

$R=\sqrt{A^{2}+B^{2}}$

In order to obtain these values, a system was developed to select the vectors during leaping. Thus, the system will select all the vectors with positive horizontal component and positive vertical component, when the subjects are leaping forward (blue arrow) by swinging their arms forward (red arrow). The resultant acceleration by both motions, which is the leaping angle as represented by green arrow (Figure7) can be calculated.

\section{RESULT AND DISCUSSION}

Angular velocity and angle of leaping were proposed in this project as the main components for distinguishing jogging and walking motions. A combined of 200 sets data from the subjects were fed into the system for analysis.

\section{A. Angular Velocity}

A range of mean values of angular velocity for walking and jogging are recorded in Table 1. From observation, the angular velocity of subjects' arms are faster in jogging than in walking. It implies that the subjects swing their arms faster in jogging than in walking. Since the range of angular velocity for walking and jogging are not overlapped with each other, this ranges can be used to recognize and differentiate both motions.

\section{B. Angle of Leaping Motion}

The second component is the angle of leaping motion of the subjects while they walk and jog. The ranges of mean of leap angle for walking and jogging were recorded in Table 1 .

Based on the table, the angle of leap in jogging is higher than in walking. This is true since in jogging motion, leaping motion do exist before the subjects get airborne. In walking motion, the angle of leaping is existed but relatively small if compared to jogging motion. The test subjects never get airborne during walking, a complete cycle of walking motion consists of two positions, they are stance (one foot is touching on the ground) and double support (both feet are touching the ground). Due to the clear difference of the ranges of leap angle between the motions, thus, it is valid to use this ranges to differentiate both of them.

\section{Differentiating Motions}

The 200 dataset (100 for jogging and 100 for walking from two subjects) was distributed into three portion, 150 for recognition analysis and 50 for testing. The recognition process using angular velocity and leaping angle was represented by the flowchart in Figure 7. A motion is classified as jogging if the angular velocity is between 2.57 $\mathrm{rad} / \mathrm{s}$ and $3.97 \mathrm{rad} / \mathrm{s}$ and the leaping angle is between $38.6^{\circ}$ and $62.31^{\circ}$. As for walking motion, it is classified if the angular velocity is between $1.10 \mathrm{rad} / \mathrm{s}$ and $1.91 \mathrm{rad} / \mathrm{s}$ and the leaping angle is between $4.63^{\circ}$ and $16.10^{\circ}$. The system will classify the data as unidentified if the detected ranges for angular velocity and leaping angle are out of these ranges.

Table. 1 Ranges of angular velocity and leaping angle of walking and jogging motions

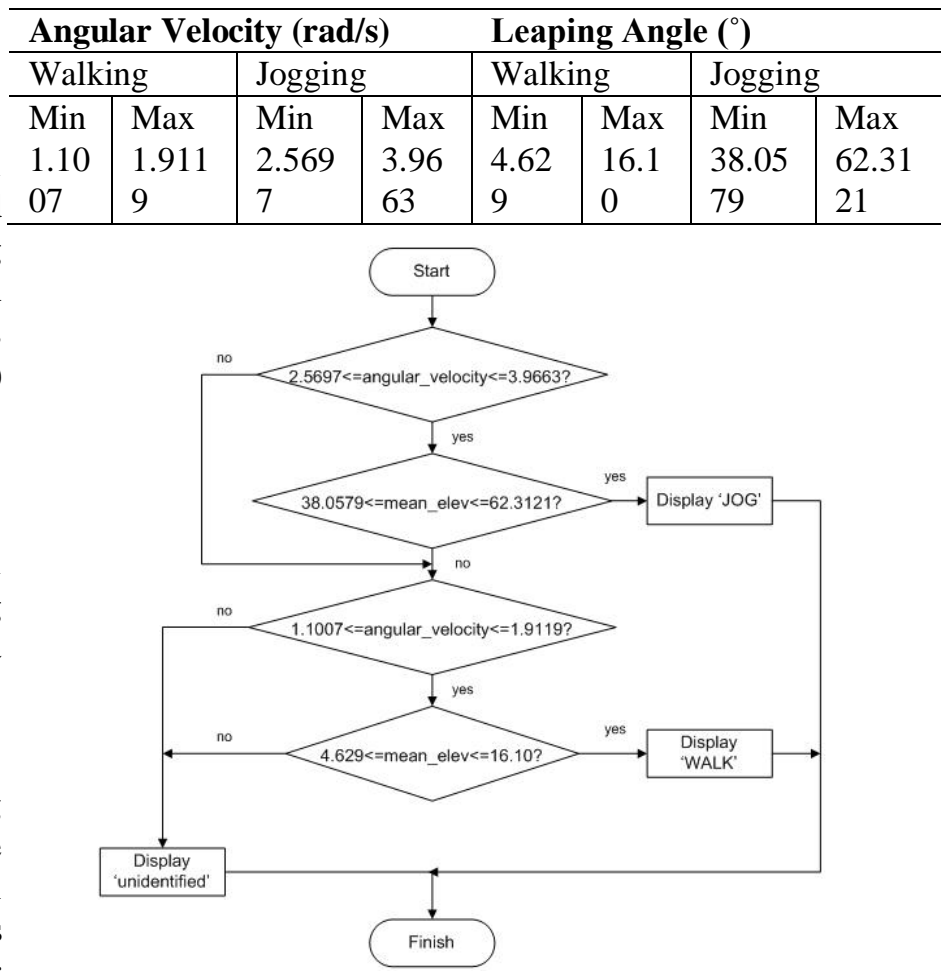

Fig. 6 Flowchart of motion classification system.

The hundred percent of the jogging motion testing data was managed to be recognized by the system, however only $70 \%$ of walking motion was accurately identified. It is due to the existence of disturbance to the movement when the subjects are performing the activities. For instance, while performing the activities on the treadmill, the subjects might miss-stepped at the side of the treadmill and causing unstable condition, therefore, the subjects might react by accelerating to catch up with the speed of the tread mill. 


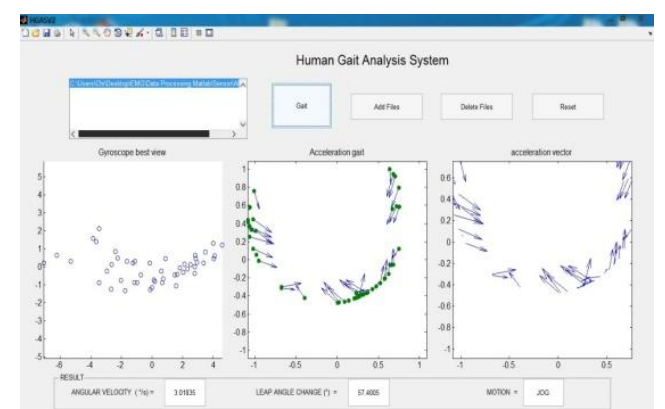

Fig. 7 Graphical User Interface of the system to recognize motion.

\section{CONCLUSION}

The 3-space sensor is useful in analyzing human motion since it consists of three different kinds of sensors in measuring the motion simultaneously. This also locate the wearable sensor based one step in front of the vision based in term of the number of different measurements that can be done simultaneously.

Scatter plot and quiver were generated to analyze human motions. Scatter plot after PCA realignment give the best view, although the variables change significantly. The quiver function in MATLAB generates the vector plot of the given motion. Thus, it gives us additional information, such as the direction of increment or decrement of the variables that we want to study.

It is also discovered that the values of angular velocity of subjects' arms and the leaping angle during walking or jogging motion can be used to differentiate human motions, with condition of the dataset must be at least 10 meter in distance. This was proved from the pilot test where 5 meter walking motion was included into the recognition process and the outcome is not positive.

In this project, a system was developed as shown in Figure7. The whole recognition process can be done by a few clicks on the mouse. The system shows the best view after PCA realignment, the acceleration gait and the acceleration vector. It also compute the values of angular velocity and leaping angle from the vectors.

\section{ACKNOWLEDGMENT}

A project of this magnitude depends on the hard work and commitment of many professionals, and we are pleased to acknowledge their contributions. The authors are deeply indebted and would like to express our gratitude to the University College of Technology Sarawak for supporting and funding this study under University Research Grant (UCTS/RESEARCH/4/2016/14).

\section{REFERENCES}

1. G. Pons-Moll, J. Romero, N. Mahmood, and M. J. Black, ACM Transactions on Graphics (TOG) 34, pp. 120, 2015

2. C.Y. Yong, S. Rubita, M. Nasrul Humaimi, and K.M. Chew, Human body and body part movement analysis using gyroscope, accelerometer and compass. In Applied Mechanics and Materials, vol. 284, pp. 31203125. Trans Tech Publications, 2013.

3. M.Shoaib, S. Bosch, O.D. Incel, H. Scholten, P.J. Havinga, Complex human activity recognition using smartphone and wrist-worn motion sensors. Sensors, 16(4), pp. 426, 2016.
4. G. Marin, F. Dominio, and P. Zanuttigh, Hand gesture recognition with jointly calibrated leap motion and depth sensor. Multimedia Tools and Applications, 75(22), pp.14991-15015, 2016.

5. W. Lu, Z. Tong, and J. Chu, Dynamic hand gesture recognition with leap motion controller. IEEE Signal Processing Letters,23(9), pp.1188-1192, 2016.

6. S. S. Rautaray and A. Agrawal, Artificial Intelligence Review 43, pp. 1-54, 2015

7. L. Bai, M. G. Pepper, Y. Yan, S. K. Spurgeon, M. Sakel, and M. Phillips, IEEE Transactions on NeuralSystems and Rehabilitation Engineering 23, 232-243, 2015.

8. A. Muro-De-La-Herran, B. Garcia-Zapirain, and A. Mendez-Zorrilla, Sensors 14, 3362-3394, 2014.

9. D. Mathias, in Staying Healthy From 1 to 100 (Springer, 2016), pp. 82-82.

10. G.Herink, D. R. Solli, M.Gulde, and C.Ropers, Field-driven photoemission from nanostructures quenches the quiver motion. Nature, 483(7388), pp.190, 2012. 